\title{
Augmented Reality X-ray Interaction in K-12 Education
}

\author{
Theory, Student Perception and Teacher Evaluation
}

\author{
Marc Ericson C. Santos, Angie Chen, Mitsuaki Terawaki, \\ Goshiro Yamamoto, Takafumi Taketomi, Jun Miyazaki, Hirokazu Kato \\ Interactive Media Design Laboratory, Graduate School of Information Science \\ Nara Institute of Science and Technology \\ Nara, Japan \\ \{chavez-s, chen-a, mitsuaki-t, goshiro, takafumi-t, miyazaki, kato\}@is.naist.jp
}

\begin{abstract}
Augmented reality (AR) $\mathrm{x}$-ray interaction is an enabling technology for providing students with virtual abstractions of the interior of an object. It provides students contextual visualization - the presentation of virtual information in the rich context of a real environment - thereby offering compelling experiences. According to experiential learning theory, such personal experiences are necessary for reaching different types of learners. AR $x$-ray is a novel interaction technique for education, thus, it is necessary to investigate how it affects the students' perception. We implemented AR x-ray using a state-of-the-art occlusion technique, and compared it to viewing 3D objects without occlusion. Results of two user studies $(n=23$ and $n=47)$ show that there are no significant differences in realism, perception of depth, and visibility with occlusion and without occlusion; and that the current technique is usable for educational purposes. We also conducted interviews with both students $(n=23)$ and teachers $(n=12)$. Results indicate that $A R x$-ray is perceived to be useful for motivating and explaining to students. The teachers expressed willingness to adopt AR x-ray and to undergo training for using AR-based teaching materials.
\end{abstract}

Keywords-augmented reality; augmented reality learning experience; $A R$ x-ray; experiential learning

\section{INTRODUCTION}

Augmented reality (AR) refers to the seamless integration of $3 \mathrm{D}$ virtual objects to real environments, made interactive in real-time [1]. The development AR's enabling technologies (software, processor, display, etc.) have led to the rise in Augmented Reality Learning Experiences or ARLEs. An increasing number of educators and technologists alike have built and evaluated prototypes. Although there are many ARLE prototypes in the current literature, there is a need for more interdisciplinary groups that will ground their work on learning theory [2]. Furthermore, it is only recently that the AR community started theorizing about the advantages of augmented reality in educational settings. One such advantage that is inherent to augmented reality is its affordance for contextual visualization: the presentation of virtual information in the rich context of a real environment. Contextual visualization enables researchers to create compelling experiences that may reach more types of learners as recommended by experiential learning theory.

We respond to the need for better understanding of ARLEs by establishing its relationship with experiential learning theory, and by proposing a novel x-ray interaction for education Section II. Then, we implemented a demonstration of the state-of-the-art AR x-ray occlusion technique, and conducted user studies with students and teachers as described in Section III. We discuss the results of the user study in Section IV. Section V concludes the paper and describes our future work.

\section{EXPERIENTIAL LEARNING AND X-RAY INTERACTION}

Experiential learning or "learning from experience" is creating meaning from our personal experiences. Experiential learning theory describes four stages (Fig. 1) in the learning cycle. Learning starts with having a concrete experience, which becomes the basis of observation and reflection. From our observations, we formulate theories, which we test for implications in new situations. Results of this testing stage provide new concrete experiences [3].

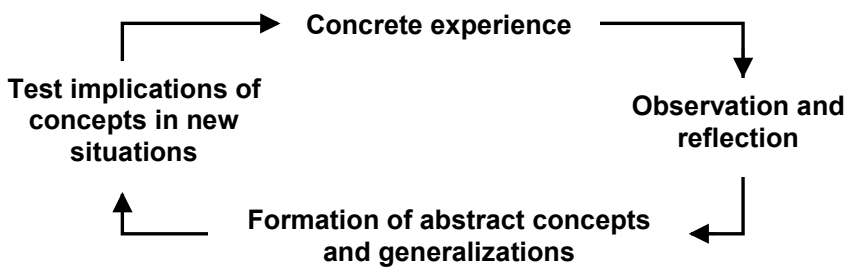

Figure 1. Lewinian Experiential Learning Model

Experiential learning requires intrinsic motivation such that learners are willing to undergo concrete experiences, and actively observe, process, and test their knowledge. Thus, for experiential learning to take place, there are two requirements: availability of the experience, and motivation on the part of the students.

Aside from topics that are suitable for laboratory experiments, many topics remain taught by conceptualization without concrete personal experiences. Some experiences are difficult to provide due to the constraints of the classroom setting such as the space, time and resources. For example, there may not be enough time and money to do fieldtrips to 
historical places, zoos, museums, etc. One type of experience that may be impractical to provide is exploring what is inside an object. For example, a student may need to look inside a person's body to observe the different organs working as a system. We propose AR x-ray interaction as a solution for observing the interior of an object.

AR x-ray is a specific kind of AR interaction wherein the user is given an illusion of looking through an occluded region, thereby exposing the actual interior of a target object [4]. The main challenge in this area is to provide sufficient occlusion cues, as such, techniques [4] and [5] are developed to decide which parts of the original scene should be kept, and which part is to be replaced by virtual overlays. The main application of AR x-ray is the visualization of what is truly inside a particular object. Applications would be visualizing the layout of sewage pipes underneath a street [5], and bodily organs during medical operations [6].

In the field of education or in designing ARLEs, we hypothesize that it is enough to visualize a virtual abstraction of the interior of a target object (i.e. we need not visualize the exact, actual interior of an object similar to the original concept of AR x-ray). Although AR x-ray cannot provide an authentic experience, it can provide experiences that are sufficient in facilitating experiential learning. As a novel interaction for education, we investigate in this paper how students perceive information using AR $\mathrm{x}$-ray.

AR $\mathrm{x}$-ray is a more compelling experience compared to printed material and purely virtual learning material because of AR's inherent advantage for contextual visualization - the presentation of virtual information in the rich context of a real environment. In AR x-ray, the real object and its accompanying environment are the "context" of the visualization, and the interior of the object (virtual 3D models and/or virtually annotated symbols) is what is being visualized. Contextual visualization leads to beneficial effects in learning because of two reasons. First, virtual information is aligned with real objects, thus reducing the need to switch attention between different media [7]. Second, multimodal cues found in a familiar, real environment are used, thus, helping the students relate and construct their knowledge [8].

$\mathrm{AR}$ has been shown to improve motivation in middleschool students based on four motivational factors: attention, relevance, confidence and satisfaction [9]. Di Serio et al. [9] observed that AR technology enhances concentration because of its immersive capabilities that help students maintain higher levels of attention. Moreover, they observed that AR interaction allows students to have more control over the exploration of content, thereby engaging different types of learners. However, AR's effect on the students' motivation to observe, process and test ideas may vary depending on the implementation of the prototypes, its features, and the delivery of the teacher. We recognize that the success of integrating AR x-ray based modules to current curriculum will depend on how the teachers will use it. As such, we interviewed teachers regarding the usability of the state-of-the-art AR x-ray, its benefits in the classroom setting, and the design issues they foresee.

\section{APPROACH}

\section{A. AR X-ray Implementation}

The AR x-ray was implemented entirely on iPad 2 tablets (dual core Apple A5, 512MB RAM, 32GB, 610 grams) using the back camera (480x640 pixel) and touch screen display (768x1024 pixel). The target real object is a cube (side $=60 \mathrm{~cm}$ ) with print on the faces as shown in Fig. 2.a. The ARToolkit [10] and fiducial markers were used to track the viewpoint and interact with the virtual object. The virtual 3D model displayed in the interior can be varied. For example, it can be a cultural artifact as shown in Fig. 2.b. The edge-based technique proposed by Sandor et al. [4] was used to combine occlusion cues from the cube to the virtual object to create an AR x-ray interaction.
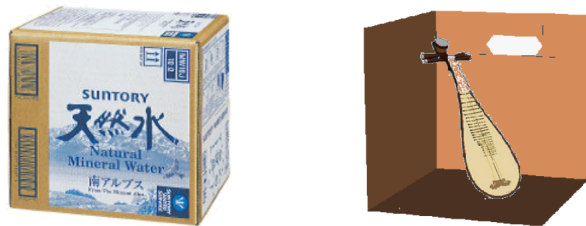

Figure 2. a. Target Object (left), b. Virtual 3D Model (right)

\section{B. User Study with Students 1}

The first user study was a pilot conducted on 23 Filipino students (9 male, 14 female, aged 5-15) attending 13 different schools ( 8 private, 5 public). Permission to join the study was obtained from the parent of the student. The study was conducted either at the house of the participant, or the house of the participant's relative or family friend. The study had three parts:

\section{1) Demonstration of $A R X$-ray}

The students were asked to point the iPad to the box and look at the box from different directions (Fig. $3)$.

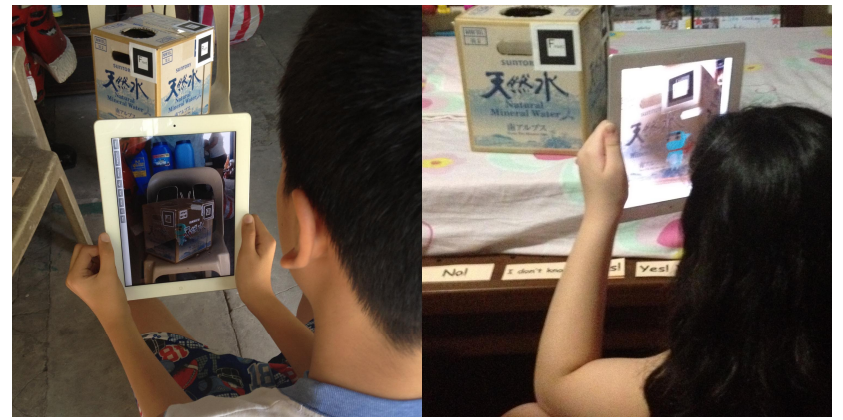

Figure 3. Participants viewing the AR x-ray demonstration.

\section{2) Perception Questionnaire}

Participants were asked to rate a five-point Likert scale on three constructs namely realism of the virtual character (Q3 and Q6), perception of depth (Q1 and Q4), and visibility of the virtual object (Q2 and Q5). 
Q1. The object is inside the box.

Q2. The object is easy to see.

Q3. The object seems real.

Q4. The object seems flat.

Q5. I can see the different parts of the object clearly.

Q6. My classmates will say the object is real.

Students were oriented about the possible answers ("No! No!," "No!," "I don't know," "Yes!" and "Yes! Yes!") which were written on 5 pieces of paper. The choices were acted out such that "No! No!" was said with strong disagreement, "No!" was said with disagreement, and so on. Then, the questions were shown, read in English, and translated to Filipino language for the students. Students then respond by pointing to one of the possible answers.

Participants were divided into two groups: control-first $(n=8)$ and experiment-first $(n=15)$. The control-first group viewed the 3D object without occlusion cues first (Fig. 4.a). Then, they answered Q1-6. After resting for five minutes, we demonstrated to them the experimental treatment having occlusion cues (AR x-ray) as shown in Fig. 4.b. Again, they answered Q1-6. We reversed the order of viewing Fig. 4.a and 4.b for the experiment-first group. The target object for viewing is a colorful ninja character to capture the participants' attention.

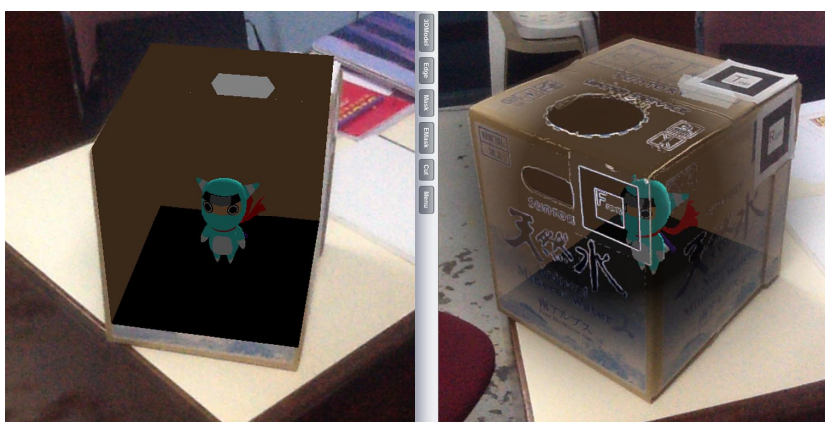

Figure 4. a. Without occlusion (left), b. With occlusion cues (right)

\section{3) Processing via Interview}

Students were engaged in a discussion to process their experience. Three questions were asked:

1. How will you describe to your classmates what you saw a while ago?

2. Are there other things you're curious to see the inside of using the magic x-ray? What are those things?

3. In school, are there some topics you have difficulty imagining and would like to use the magic $\mathrm{x}$-ray on? Which topics are those?

\section{User Study with Students 2}

The second user study was conducted on 47 students (21 male, 26 female, aged 11-16). All participants attend Spring Christian School in Muntinlupa City, Philippines. The user study was conducted inside the school campus. A similar process as "User Study with Students 1" was used but we omitted the third part, "processing via interview," because the students had limited time.

Instead of reading and translating Q1-6 to each student, questionnaires were used to facilitate faster gathering of data. The 47 students were divided into two groups: control-only $(n=21)$ and experiment-only $(n=26)$. The control-only group viewed the object without occlusion (Fig. 4.a), then they answered the questionnaire. On the other hand, the experiment-only group viewed the object with the occlusion cues (Fig. 4.b), then they answered the questionnaire.

\section{Interview with Teachers}

Six teachers from Spring Christian School and another six teachers from Spurgeon School in Makati City, Philippines (5 male, 7 female, aged 20-54) participated in this study. AR x-ray was demonstrated, and the definition of AR and AR x-ray interaction was explained to the teachers. We then ask them the following questions:

1. Do you think AR x-ray is useful for teaching?

2. Which topics do you imagine AR x-ray to be necessary?

3. What would be the advantages and disadvantages of using AR x-ray to teach these topics?

Sketches of two possible applications were presented to the teachers. The first one is for looking inside the body to see the skeletal system [11]. The second one is looking inside plants to see how water is transported. Teachers were then asked for advantages, disadvantages and suggestions on these application sketches.

\section{RESUlTS AND DisCUSSION}

\section{A. Students Perception of Realism, Depth and Visibility}

We used two questions each to represent the three constructs: realism, depth and visibility. Responses were mapped into scores such that "No! No!" is 1 and "Yes! Yes!" is 5. Aside from the fourth question (Q4), higher scores would correspond to higher perception of the construct. We converted Q4 by subtracting it from 6, to get a score that would correspond to a higher perception of depth. That is, we assume that if a participant reported "No!" when asked, "The object seems flat;" then he would have reported "Yes!" if asked, "The object is NOT flat."

Table I shows the correlation of the responses of participants to the pair of questions representing the three constructs. Questions 1 and 4 have no correlation in the first user study and a negative correlation in the second user study. Students tend to disagree that the object seems flat (or agree that it is not flat). However, depending on the interpretation of the student, the answer to whether or not 
the object is inside the box varies. According to the participants, when they disagreed that the object is inside the box, they meant that the object is not actually inside the box and that it is only an illusion. Thus, Q1 is not a reliable measure for the perception of depth. The other two pairs of questions received moderately consistent responses from the participants.

TABLE I. CORRELATION OF SCORES FOR QUESTIONS REPRESENTING REALISM, DEPTH AND VISIBILITY

\begin{tabular}{|l|c|c|}
\hline Constructs (Questions) & User Study 1 (n=23) & User Study 2 (n=47) \\
\hline Realism (Q3 and Q6) & 0.27 & 0.44 \\
\hline Depth (Q1 and Q4) & 0.03 & -0.39 \\
\hline Visibility (Q2 and Q5) & 0.65 & 0.56 \\
\hline
\end{tabular}

We conducted an unpaired t-test on the 6 questions measuring realism, depth and visibility. Tables II and III summarize the mean, $95 \%$ confidence interval for the mean, and standard deviation for user study 1 and 2 , respectively.

TABLE II. MEAN AND STANDARD DEVIATION OF CONTROL AND EXPERIMENTAL SCENARIOS OF USER STUDY

\begin{tabular}{|c|c|c|c|c|}
\hline \multirow[b]{2}{*}{$\begin{array}{l}\text { Question } \\
\text { (Construct) }\end{array}$} & \multicolumn{2}{|c|}{ Without Occlusion } & \multicolumn{2}{|c|}{ With Occlusion (x-ray) } \\
\hline & $\begin{array}{c}\text { Mean } \\
\text { (95\% Conf.) } \\
\end{array}$ & STDV & $\begin{array}{c}\text { Mean } \\
\text { (95\% Conf.) } \\
\end{array}$ & STDV \\
\hline Q1 (Depth) & $3.7(3.1-4.2)$ & 1.2 & $3.6(3.1-4.1)$ & 1.3 \\
\hline Q2 (Visibility) & $4.0(3.5-4.4)$ & 1.0 & $3.3(2.8-3.7)$ & 1.2 \\
\hline Q3 (Realism) & $3.4(2.8-3.9)$ & 1.4 & $2.7(2.2-3.3)$ & 1.3 \\
\hline Q4 (Depth) & $4.1(3.7-4.6)$ & 0.9 & $4.0(3.5-4.4)$ & 1.2 \\
\hline Q5 (Visibility) & $4.0(3.6-4.5)$ & 0.9 & $3.6(3.1-4.1)$ & 1.3 \\
\hline Q6 (Realism) & $3.3(2.8-3.8)$ & 1.2 & $3.1(2.6-3.7)$ & 1.3 \\
\hline
\end{tabular}

TABLE III. MEAN AND STANDARD DEVIATION OF CONTROL AND EXPERIMENTAL SCENARIOS OF USER STUDY 2

\begin{tabular}{|l|c|c|c|c|}
\hline \multirow{2}{*}{$\begin{array}{c}\text { Question } \\
\text { (Construct) }\end{array}$} & \multicolumn{2}{|c|}{ Without Occlusion } & \multicolumn{2}{c|}{ With Occlusion (x-ray) } \\
\cline { 2 - 5 } & $\begin{array}{c}\text { Mean } \\
\text { (95\% Conf.) }\end{array}$ & STDV & $\begin{array}{c}\text { Mean } \\
\text { (95\% Conf.) }\end{array}$ & STDV \\
\hline Q1 (Depth) & $3.4(2.9-3.9)$ & 1.2 & $3.4(3.0-3.9)$ & 1.2 \\
\hline Q2 (Visibility) & $3.8(3.3-4.3)$ & 1.1 & $4.0(3.5-4.4)$ & 1.1 \\
\hline Q3 (Realism) & $3.1(2.6-3.7)$ & 1.2 & $3.0(2.5-3.5)$ & 1.3 \\
\hline Q4 (Depth) & $3.3(2.9-3.7)$ & 1.0 & $3.3(2.9-3.7)$ & 1.0 \\
\hline Q5 (Visibility) & $4.0(3.5-4.5)$ & 0.6 & $3.7(3.2-4.1)$ & 1.0 \\
\hline Q6 (Realism) & $3.4(3.0-3.9)$ & 1.0 & $3.7(3.4-4.2)$ & 1.0 \\
\hline
\end{tabular}

Results show that there are no significant differences between viewing virtual $3 D$ objects with or without occlusion cues. In Tables II and III, majority of the mean and standard deviation for with and without occlusion are almost equal. Based on the unpaired t-tests, only Q2 in User Study 1 indicates a significant difference $(p$-value $=0.041)$. In the first user study, the participants report that they are able to see the object more easily when there are no occlusion cues (score $=4.0$ ) than when occlusion cues are added (score $=3.3$ ). Participants explained that the object is less easy to see when there are occlusion cues because "there is a barrier." However, this significant difference was not observed in Q2 in User Study $2(\mathrm{p}$-value $=0.65)$. Thus, we have no repeated proof to reject the null hypothesis: There are no significant differences in realism, perception of depth, and visibility when viewing virtual $3 D$ objects with or without occlusion cues.

\section{B. Students' Initial Understanding of AR and AR X-ray}

Discussions with the 23 participants of User Study 1 reveal that K-12 students think of AR x-ray as the addition, placement or appearance of a virtual object. Although 16 of the participants are familiar with video games in various devices, only 4 of them described AR x-ray as 3D, graphics or effects. Students tend to attach AR x-ray to the use of a tablet computer. They tend to say, "I can see the object in the iPad," and "I can only see the object through the iPad."

AR x-ray did not lead to confusion while the students were using it. However, when they start reflecting on the experience, some students feel the need to confirm whether or not the virtual object is really inside the box. They want to look inside the box to know what is trully inside. In the case of a box, it is easy to open it and show to the students. However, in future use cases of AR x-ray interaction, opening the target object may not be practical. This is a limitation of AR x-ray interaction, and may affect its application in experiential learning. The student's concrete experience is mediated by a device (iPad), and the student may have difficulty trusting the information presented to him via a novel AR x-ray interaction.

\section{Teachers' Evaluation of AR X-ray}

Teachers expressed their interest in using AR x-ray learning materials, and their willingness to undergo training for using "high technology" learning materials. The teachers believe that some topics are easier to explain to students when using AR x-ray. Motivated by possible improvements in student learning, they are willing to use AR x-ray learning materials, given that the materials are available and training is provided. According to teachers, they regularly update their skill set by attending seminars and workshops, which can be a venue for learning new types of learning materials.

The teachers identified three advantages of AR x-ray in their current practice:

1. Learning by experience - Eight of the teachers identified "learning by experience" as an advantage of AR x-ray. Aside from the current book-based illustrations, students are given another kind of experience that catches their attention and motivates them to study more.

2. Improved attention - All the teachers said that AR $\mathrm{X}$-ray will help generate interest among students because they believe that most of their students are visual learners, and are accustomed with new gadgets and video games.

3. Increased motivation - AR x-ray motivates students by first catching their attention and making them curious to know more. The teachers believe that AR $\mathrm{x}$-ray does not replace teachers or books. Instead, 
AR x-ray can lead the students to ask more questions while in class, and read more from their books.

The teachers outline three key issues that must be addressed to have a successful integration of AR x-ray learning materials in their current practice.

1. Cost of technology and availability of devices for students - The school must be able to afford the hardware, software and teacher training requirements of the AR x-ray interaction. Moreover, it is better if each student is provided with a device, instead of many students sharing one device. In the Philippines, some private schools have already adapted tablet computers to replace textbooks.

2. Accuracy of virtual information - Teachers pointed out that AR x-ray is highly subjected to misinformation. The virtual abstraction provided by AR x-ray may be inaccurate. For example, in displaying body organs inside a student, the size of organs and their relative positions will vary depending on the body type. Inaccurate size and positioning of organs can misinform students with the size and location of their body organs.

3. Time constraint - AR x-ray and other augmented reality learning experience can be highly interactive. As such, learning modules using AR x-ray must be carefully planned to work with the original time frame allotted for the topic.

\section{CONCLUSION AND FUTURE WORK}

We introduced AR x-ray as one of the interactions for augmented reality learning experiences (ARLEs). AR x-ray in education is different from the original use case which is to display the actual interior of an object. In educational settings, we hypothesize that it is sufficient to provide virtual abstractions of the inside of an object to create experiences for experiential learning. AR x-ray provides a more compelling experience compared to printed material and purely virtual learning material because of AR's inherent advantage for contextual visualization - the presentation of virtual information in the rich context of a real environment. In the case of AR x-ray, the real object and its accompanying environment are the context of the visualization, and the interior of the object (virtual 3D models and/or virtually annotated symbols) is what is being visualized.

As a novel interaction, we investigated how students' perceive realism, depth and visibility in two user studies. Results show that there are no significant differences between viewing a regular 3D object, compared to viewing the 3D object with occlusion cues. Therefore, the state-ofthe-art occlusion technique is as realistic and as easy to visualize as $3 \mathrm{D}$ computer graphics that students are familiar with in video games. We conclude that the state-of-the-art occlusion technique is sufficient to create AR x-ray interaction for ARLEs.
Discussions with students reveal that they are confident to describe AR x-ray with their own vocabulary. There was no confusion when the students were using x-ray AR. However, students feel the need to actually see inside the box to confirm their understanding. This may not be practical in future applications of AR x-ray, and becomes a limitation of the experience provided by AR X-ray learning materials.

Teachers are interested in using AR x-ray because they believe that it gives the student a chance to learn by experience. They believe it will generate interest in the students that may lead them to wanting to learn more by asking questions and reading textbooks. AR x-ray learning materials must be made as accurate as possible to prevent misinformation. It must also adjust to the available resources for teaching especially in terms of budget and time.

In the future, we will be implementing ARLEs that uses AR x-ray interaction. We will then conduct user studies on these ARLEs by asking teachers to use it in their class. We are interested in observing how AR x-ray affects constructs such as: motivation, student's trust, and student's perception.

\section{ACKNOWLEDGMENT}

The authors would like to thank Michael Daniel Austria, Spring Christian School, and Surgeon School for their cooperation in the user studies.

\section{REFERENCES}

[1] R. Azuma, "A survey of augmented reality," Presence-Teleoperators and Virtual Environments, vol. 6, no. 4, 1997, pp. 355-385.

[2] M. Billinghurst and A. Duenser. "Augmented Reality in the Classroom," Computer Magazine, vol. 45, no. 7, 2012, pp. 56-63.

[3] D. A. Kolb, "Experiential learning: experience as the source of learning and development," Prentice Hall, 1984, p. 21.

[4] C. Sandor, A. Cunningham, A. Dey and V. Mattila. "An Augmented Reality X-Ray System Based on Visual Saliency," Proc. IEEE Int'l Symp. Mixed and Augmented Reality, October 2010, pp. 27-36.

[5] S. Zollmann, D. Kalkofen, E. Mendez and G. Reytmayr. "Imagebased Ghostings for Single Layer Occlusions in Augmented Realiy," Proc. IEEE Int'l Symp. on Mixed and Augmented Reality Proceedings, pp. 19-26, October 2010.

[6] Navab, N., Blum, T., Wang, L., Okur, A. and Wendler, T. First Deployments of Augmented Reality in Operating Rooms, In IEEE Computer Magazine, 45, 7, pp.48-55, 2012.

[7] I. Radu, "Why Should My Students Use AR: A Comparative Review of the Educational Impacts of Augmented-Reality." Proc. IEEE Int'l Symp. on Mixed and Augmented Reality, pp. 313-314, November 2012.

[8] W. Tarng. and K. A. Ou, "Study of Campus Butterfly Ecology Learning System Based on Augmented Reality and Mobile Learning," Proc. IEEE Conf. Wireless, Mobile and Ubiquitous Technology in Education, pp. 62-66, 2012.

[9] A. Di Serio, M. B. Ibáñez, and C. D. Kloos. "Impact of an Augmented Reality System on Students' Motivation for a Visual Art Course." Computers \& Education, 2012.

[10] Kato, H. and Billinghurst, M. Marker Tracking and HMD Calibration for a video-based Augmented Reality Conferencing System, In International Workshop in Augmented Reality Proceedings, 1999.

[11] T. Blum, V. Kleeberger, C. Bichlmeier, and N. Navab. "mirracle: An augmented reality magic mirror system for anatomy education." IEEE Virtual Reality Workshops, pp. 115-116, 2012. 